\title{
Tecnis Symfony Intraocular Lens with a "Sweet Spot" for Tolerance to Postoperative Residual Refractive Errors
}

\author{
Béatrice Cochener \\ Ophthalmology Department, Brest University Hospital, Brest, France \\ Email: beatrice.cochener@ophtalmologie-chu29.fr
}

How to cite this paper: Cochener, B. (2017) Tecnis Symfony Intraocular Lens with a "Sweet Spot" for Tolerance to Postoperative Residual Refractive Errors. Open Journal of Ophthalmology, 7, 14-20. https://doi.org/10.4236/ojoph.2017.71003

Received: January 2, 2017

Accepted: January 31, 2017

Published: February 3, 2017

Copyright $\odot 2017$ by author and Scientific Research Publishing Inc. This work is licensed under the Creative Commons Attribution International License (CC BY 4.0).

http://creativecommons.org/licenses/by/4.0/

\section{(c) (i) Open Access}

\begin{abstract}
Purpose: To investigate the impact of residual astigmatism on visual acuity (VA) after the implantation of a novel extended range of vision (ERV) intraocular lens (IOL) based on the correction of spherical and chromatic aberration. Method: The study enrolled 411 patients bilaterally implanted with the ERV IOL Tecnis Symfony. Visual acuity and subjective refraction were analyzed during the 4- to 6-month follow-up. The sample of eyes was stratified for four groups according to the magnitude of postoperative refractive astigmatism and postoperative spherical equivalent. Results: The astigmatism analysis included 386 eyes of 193 patients with both eyes of each patient within the same cylinder range. Uncorrected VAs for distance, intermediate and near were better in the group of eyes with lower level of postoperative astigmatism, but even in eyes with residual cylinders up to $0.75 \mathrm{D}$, the loss of VA lines was clinically not relevant. The orientation of astigmatism did not seem to have an impact on the tolerance to the residual cylinder. The SE evaluation included 810 eyes of 405 patients, with both eyes of each patient in the same SE range. Uncorrected VAs for distance, intermediate and near, were very similar in all SE groups. Conclusion: Residual cylinders up to $0.75 \mathrm{D}$ after the implantation of the Tecnis Symfony IOL have a very mild impact on monocular and binocular VA. The Tecnis Symfony IOL shows a good tolerance to unexpected refractive surprises and thus a better "sweet spot".
\end{abstract}

\section{Keywords}

Extended Range of Vision IOL, Astigmatism Tolerance, Refractive Tolerance, Tecnis Symfony

\section{Introduction}

At present, multifocal intraocular lenses (MF IOLs) appear to provide the most 
consistent and reliable method for the surgical correction of presbyopia [1]. However, residual astigmatism is one of the leading causes of dissatisfaction after the implantation of a MF IOL [2]. These IOLs require emmetropia for the achievement of the best visual outcomes, and small amounts of astigmatism may limit visual performance significantly [3]. Thus, astigmatism has to be completely corrected in order to obtain the maximum efficiency of a MF IOL [4]. The rate of enhancement to reduce residual astigmatism after cataract surgery with implantation of a MF IOL ranges from $5.24 \%$ to $23.66 \%$ depending on the study. Specifically, Gundersen et al. reported a significant rate of retreatments after MF IOL implantation (10.8\%) over a sample of 416 eyes, most of which are due to reduced visual acuity (VA) secondary to residual astigmatism. These authors found no statistically significant differences in the impact of residual astigmatism on the visual outcome between different traditional MF IOL models [5]. Femtosecond laser-assisted procedures have been proposed as a valid alternative for correcting residual refractive errors after cataract surgery [6] [7]. However, the well-known potential complications of corneal refractive procedures, including the induction of higher-order aberrations (HOAs), and the secondary degradation of the retinal image may also lead to dissatisfaction. Current research is aimed at developing optical designs with a higher tolerance to postoperative defocus. This paper reports our experience about the impact of residual astigmatism on VA after the implantation of a novel extended range of vision (ERV) IOL based on the correction of spherical and chromatic aberration.

\section{Patients and Methods}

A prospective international multicenter study, the CONCERTO study, was designed to evaluate the clinical outcomes obtained after the implantation of the Tecnis Symfony ZRX00 ERV IOL (Abbott Medical Optics, Santa Ana, USA). The Tecnis Symfony IOL has an achromatic diffractive pattern that elongates the range of vision of the eye and compensates for the chromatic and spherical aberration of the cornea. Specifically, the lens has a biconvex wavefront-designed anterior aspheric surface and a posterior achromatic diffractive surface. The total diameter of the IOL is $13 \mathrm{~mm}$ and the optic zone diameter is $6.0 \mathrm{~mm}$. It is made of a UV-blocking hydrophobic acrylic material, with a refractive index of 1.47 at $35^{\circ} \mathrm{C}$.

This research included patients who had undergone cataract surgery or clear lens extraction with bilateral implantation of the mentioned ERV IOL. Exclusion criteria included previous ocular surgery, chronic or recurrent uveitis, acute ocular disease or external/internal infection, diabetes with retinal changes, glaucoma or intraocular pressure (IOP) equal or higher than $24 \mathrm{mmHg}$, pseudoexfoliation syndrome, pathological miosis, keratoconus, and corneal endothelial dystrophy. All patients were informed about the study and provided informed consent to undergo the clinical examinations in accordance with the tenets of the Declaration of Helsinki.

The last preoperative examination of the patients included measurement of 
monocular and binocular uncorrected distance (UDVA), intermediate (UIVA), and near visual acuity (UNVA), monocular and binocular corrected distance visual acuity (CDVA), distance-corrected intermediate (DCIVA) and distancecorrected near visual acuity (DCNVA), optical biometry, manifest refraction, biomicroscopy, Goldmann applanation tonometry, and fundoscopy. Postoperatively, all patients were evaluated during a 4 - 6-months follow-up. Monocular and binocular UDVA and CDVA, and binocular UIVA and UNVA, as well as subjective refraction were assessed at the end of the follow-up.

For the analyses, the sample of eyes was subdivided into four main groups according to the level of postoperative refractive astigmatism: less than $0.25 \mathrm{D}, 0.25$ to $<0.5 \mathrm{D}, 0.5$ to $<0.75 \mathrm{D}$ cylinder, and more than $0.75 \mathrm{D}$. Another subdivision was also performed considering the postoperative spherical equivalent (SE): within $\pm 0.25 \mathrm{D}$, within $\pm 0.50 \mathrm{D}$, within $\pm 0.75 \mathrm{D}$, within $\pm 1.00 \mathrm{D}$, and within \pm 2.00 D. In order to evaluate the binocular visual acuity measurements, patients were only included in this analysis if both eyes were in the same postoperative cylinder range or within the same range of postoperative spherical equivalent, respectively.

SPSS statistics software package version 15.0 for Windows (IBM, Armonk, NY, USA) was used for the analyses. Mean values of binocular UDVA, UIVA and UNVA were obtained with their corresponding standard deviation values. Differences between the impacts of the astigmatism orientation were analysed with the Mann-Whitney test.

\section{Results}

\section{Postoperative cylinder}

A total of 386 eyes of 193 patients were included in this evaluation with both eyes of each patient within the same cylinder range. A total of 184 eyes of $92 \mathrm{pa}$ tients $(47.67 \%$ of the sample) showed a postoperative astigmatism equal or higher than $0.50 \mathrm{D}$, while 202 eyes of 101 patients (52.33\% of the sample) had a postoperative cylinder of less than $0.50 \mathrm{D}$. The demographic data of the groups are shown in Table 1 . There were no statistically significant differences regarding demographic data between groups, except for the proportion of females and males.

Table 2 shows the monocular and binocular UDVA and CDVA outcomes, as well as binocular UIVA and UNVA data for the four subgroups stratified according to the residual postoperative astigmatism. As shown, uncorrected VAs for distance, intermediate and near were better in the group of eyes with lower

Table 1. Demographic data in the 4 groups stratified for postoperative residual cylinder.

\begin{tabular}{ccccc}
\hline & \multicolumn{4}{c}{ Postoperative residual cylinder } \\
\cline { 2 - 5 } & $\mathbf{0}$ to $<\mathbf{0 . 2 5} \mathrm{D}$ & $\mathbf{0 . 2 5}$ to $<0.5 \mathrm{D}$ & $\mathbf{0 . 5}$ to $<0.75 \mathrm{D}$ & $\mathbf{2 0 . 7 5} \mathrm{D}$ \\
\hline No. of patients & 91 & 10 & 42 & 50 \\
Age (mean \pm SD) & $63.3 \pm 9.1$ & $65.2 \pm 10.0$ & $63.1 \pm 12.03$ & $69.88 \pm 10.8$ \\
Gender female/male (\%) & $68.1 / 31.9$ & $70.0 / 30.0$ & $59.5 / 40.5$ & $46.0 / 54.0$ \\
\hline
\end{tabular}


level of postoperative astigmatism (Table 2). However, even in the group of eyes with residual cylinders up to $0.75 \mathrm{D}$, the loss of VA lines was not clinically relevant. Specifically, binocular UDVA only suffered a slight reduction of 1 line for a postoperative astigmatism up to $0.75 \mathrm{D}$. Likewise, binocular UIVA and UNVA changed from 0.89 to 0.81 and from 0.72 to 0.70 with surgery, respectively.

The orientation of astigmatism did not seem to have an impact on the tolerance to the residual cylinder, as shown in Figure 1. No significant differences in binocular UDVA, UIVA and UNVA were found among eyes with with-the-rule (WTR), against-the-rule (ATR) and oblique (OBL) astigmatism ( $\mathrm{p} \geq 0.143)$. In any case, it should be mentioned that significant differences were found in the magnitude of sphere and cylinder among eyes with with-the-rule (WTR), against-the-rule (ATR) and oblique (OBL) astigmatism (Figure 2). Specifically, significantly lower magnitude of cylinder and significantly higher magnitude of sphere were found in the groups of eyes with ATR astigmatism compared to those with WTR and OBL astigmatisms $(\mathrm{p}<0.001)$.

\section{Postoperative spherical equivalent}

Besides the impact of residual cylinder, the tolerance of the Tecnis Symfony IOL to the level of postoperative SE was also evaluated. In this case, the evaluated sample included a total of 810 eyes of 405 patients, with both eyes of each patient in the same SE range. The demographic data of the groups are shown in

Table 2. Influence of the residual cylinder on decimal visual acuity (4 - 6 months, CONCERTO Study). Abbreviations: UDVA (Uncorrected Distance Visual Acuity); CDVA (Corrected Distance Visual Acuity); UIVA (Uncorrected Intermediate Visual Acuity); UNVA (Uncorrected Near Visual Acuity); OD (right eye); OS (left eye).

\begin{tabular}{|c|c|c|c|c|c|c|c|c|c|}
\hline Cylinder range (D) & UDVA OD & UDVA OS & UDVA OU & CDVA OD & CDVA OS & CDVA OU & UIVA OU & UNVA OU & $\mathrm{N}$ \\
\hline 0 to $<0.25$ & $0.90 \pm 0.27$ & $0.89 \pm 0.20$ & $1.02 \pm$ & 1.02 & $1.01=$ & 1.09 & 0.89 & 0.72 & 91 \\
\hline 0.25 to $<0.5$ & $0.82 \pm 0.22$ & $0.85 \pm 0.24$ & $1.00 \pm 0.23$ & $0.99 \pm 0.12$ & $0.99 \pm 0.12$ & $1.18 \pm 0.31$ & $0.68 \pm 0.28$ & $0.54 \pm 0.18$ & 10 \\
\hline 0.5 to $<0.75$ & $0.78 \pm 0.21$ & $0.83 \pm 0.20$ & $0.90 \pm 0.17$ & $0.97 \pm 0.17$ & $0.96 \pm 0.17$ & $1.01 \pm 0.18$ & $0.81 \pm 0.28$ & $0.70 \pm 0.24$ & 42 \\
\hline 0.75 and more & $0.74 \pm 0.23$ & $0.72 \pm 0.23$ & $0.84 \pm 0.20$ & $0.95 \pm 0.15$ & $0.94 \pm 0.17$ & $0.99 \pm 0.16$ & $0.72 \pm 0.24$ & $0.63 \pm 0.24$ & 50 \\
\hline
\end{tabular}

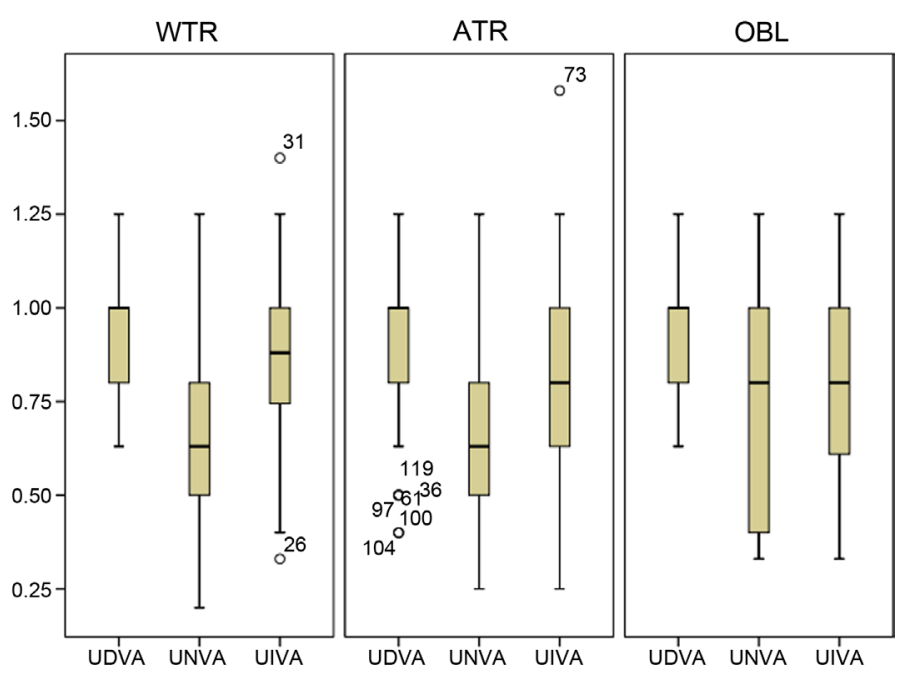

Figure 1. Distribution of the binocular visual outcome according to the orientation of astigmatism (WTR: with the rule, ATR: against the rule, OBL: oblique). 
Table 3. There were no statistically significant differences regarding demographic data between groups.

Table 4 shows the monocular and binocular UDVA and CDVA outcomes, as well as binocular UIVA and UNVA data for the five subgroups stratified according to the residual postoperative SE. Uncorrected distance, intermediate and near VAs were very similar in all SE groups (Table 4). Binocular UDVA did not change significantly and only showed a very slight difference of 0.05 between a postoperative SE of \pm 0.25 and \pm 2.0 D. Likewise, the levels of uncorrected binocular intermediate and near VAs improved slightly for higher magnitudes of residual SE.
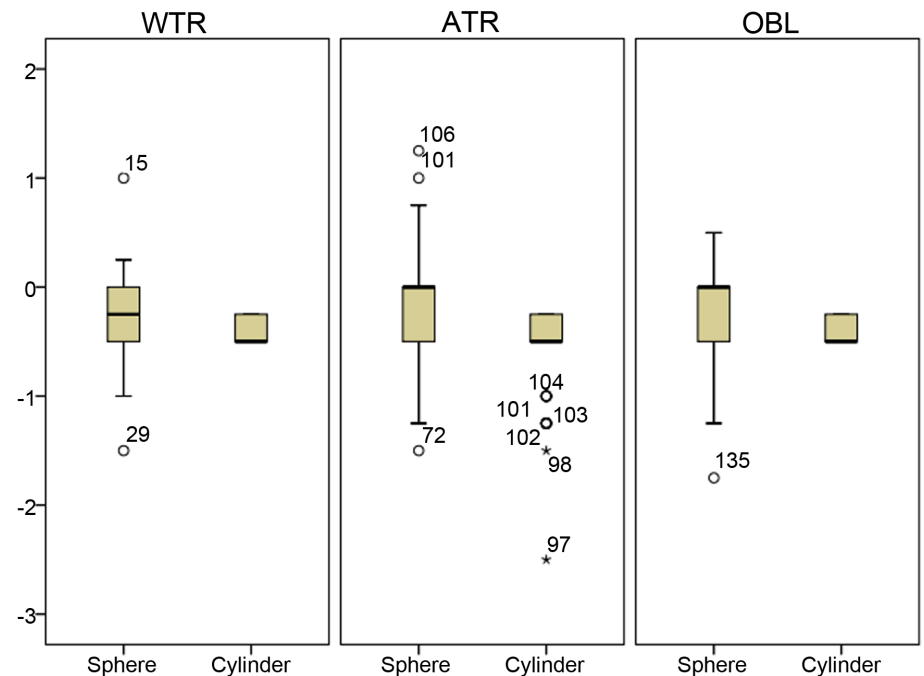

Figure 2. Magnitude of sphere and cylinder in eyes with different orientation of astigmatism (WTR: with the rule, ATR: against the rule, OBL: oblique).

Table 3. Demographic data in the 4 groups stratified for postoperative spherical equivalent.

\begin{tabular}{cccccc}
\hline & \multicolumn{5}{c}{ Postoperative spherical equivalent } \\
\cline { 2 - 6 } & within $\mathbf{\pm 0 . 2 5}$ & within $\mathbf{\pm 0 . 5}$ & within $\mathbf{\pm 0 . 7 5}$ & within $\pm \mathbf{1 . 0}$ & within $\pm \mathbf{2 . 0}$ \\
\hline No. of patients & 134 & 229 & 307 & 359 & 405 \\
Age (mean \pm SD) & $63.1 \pm 11.2$ & $65.5 \pm 10.9$ & $65.3 \pm 10.7$ & $64.6 \pm 10.9$ & $65.5 \pm 10.9$ \\
Gender: female/male (\%) & $61.9 / 38.1$ & $57.2 / 42.8$ & $58.6 / 41.1$ & $59.3 / 40.7$ & $59.8 / 40.26$ \\
\hline
\end{tabular}

Table 4. Influence of residual spherical equivalent on visual acuity (4 - 6 months, CONCERTO Study). Abbreviations: SE (Spherical Equivalent); UDVA (Uncorrected Distance Visual Acuity); CDVA (Corrected Distance Visual Acuity); UIVA (Uncorrected Intermediate Visual Acuity); UNVA (Uncorrected Near Visual Acuity); OD (right eye); OS (left eye).

\begin{tabular}{|c|c|c|c|c|c|c|c|c|c|}
\hline SE range (D) & UDVA OD & UDVA OS & UDVA OU & CDVA OD & CDVA OS & CDVA OU & UIVA OU & UNVA OU & $\mathbf{N}$ \\
\hline Within \pm 0.25 & $0.92 \pm 0.17$ & $0.92 \pm 0.19$ & $1.00 \pm 0.15$ & $0.98 \pm 0.15$ & $0.99 \pm 0.18$ & $1.06 \pm 0.18$ & $0.77 \pm 0.26$ & $0.62 \pm 0.22$ & 134 \\
\hline Within \pm 0.50 & $0.90 \pm 0.19$ & $0.89 \pm 0.19$ & $0.99 \pm 0.17$ & $0.98 \pm 0.15$ & $0.99 \pm 0.17$ & $1.06 \pm 0.18$ & $0.79 \pm 0.26$ & $0.63 \pm 0.22$ & 229 \\
\hline Within \pm 0.75 & $0.87 \pm 0.20$ & $0.87 \pm 0.19$ & $0.98 \pm 0.18$ & $0.99 \pm 0.15$ & $0.99 \pm 0.16$ & $1.06 \pm 0.18$ & $0.81 \pm 0.27$ & $0.65 \pm 0.23$ & 307 \\
\hline Within \pm 1.00 & $0.84 \pm 0.22$ & $0.85 \pm 0.21$ & $0.96 \pm 0.19$ & $0.99 \pm 0.16$ & $0.98 \pm 0.16$ & $1.06 \pm 0.18$ & $0.80 \pm 0.27$ & $0.67 \pm 0.24$ & 359 \\
\hline Within \pm 2.00 & $0.82 \pm 0.23$ & $0.84 \pm 0.22$ & $0.95 \pm 0.20$ & $0.98 \pm 0.16$ & $0.98 \pm 0.16$ & $1.05 \pm 0.18$ & $0.81 \pm 0.27$ & $0.68 \pm 0.25$ & 405 \\
\hline
\end{tabular}




\section{Discussion}

This observational type of study enrolled patients with the need or desire of lens exchange. In order to be able to evaluate the impact of the IOL implantation on residual astigmatism or spherical equivalent, the exclusion criteria were chosen in a way that other ocular pathologies don't blur the refractive results.

In the CONCERTO study, the Tecnis Symfony IOL provided a homogeneous and excellent visual restoration across all distances after cataract surgery or clear lens exchange, with minimal levels of disturbing photic phenomena [8]. Previous theoretical and in-vitro studies have demonstrated that the combination of the compensation for chromatic aberration, primary spherical aberration and unique echellette design results in an increased ability to provide an extended range of vision [9] [10] [11]. Also, the light distribution of the Tecnis Symfony IOL between its best vision foci has been demonstrated to be more homogeneous and less vergence dependent under dim conditions compared to multifocal IOLs [12]. This special optical design allows excellent optical and visual outcomes for far and intermediate distances, and a functional range of VA for near.

According to our results, residual cylinders up to $0.75 \mathrm{D}$ after the implantation of the Tecnis Symfony IOL have a very mild and clinically insignificant impact on monocular and binocular UDVA. Likewise, binocular UIVA and UNVA are not significantly affected in this situation. The orientation of the residual astigmatism does not seem to have either a significant impact on the visual outcome achieved with the Tecnis Symfony IOL, with similar UDVA, UIVA and UNVA in eyes with WTR, ATR and OBL astigmatisms. Regarding the postoperative SE, the Tecnis Symfony IOL shows a good tolerance to unexpected refractive surprises and thus a better "sweet spot".

\section{Conclusion}

In summary, the Tecnis Symfony IOL is a new promising alternative to provide an effective and continuous range of vision from far to near distances after cataract surgery or refractive lens exchange. This novel IOL provides an added value by the good tolerance to postoperative residual refractive errors, which is a key factor for ensuring patient satisfaction.

\section{References}

[1] Charman, W.N. (2014) Developments in the Correction of Presbyopia II: Surgical Approaches. Ophthalmic and Physiological Optics, 34, 397-426. https://doi.org/10.1111/opo.12129

[2] De Vries, N.E., Webers, C.A., Touwslager, W.R., Bauer, N.J., De Brabander, J., Berendschot, T.T. and Nuijts, R.M. (2011) Dissatisfaction after Implantation of Multifocal Intraocular Lenses. Journal of Cataract \& Refractive Surgery, 37, 859865. https://doi.org/10.1016/j.jcrs.2010.11.032

[3] Macsai, M.S. and Fontes, B.M. (2008) Refractive Enhancement Following Presbyopia-Correcting Intraocular Lens Implantation. Current Opinion in Ophthalmology, 19, 18-21. https://doi.org/10.1097/ICU.0b013e3282f14d9f

[4] Abdelghany, A.A. and Alió, J.L. (2014) Surgical Options for Correction of Refractive 
Error Following Cataract Surgery. Eye Vis (London), 1, 2. https://doi.org/10.1186/s40662-014-0002-2

[5] Gundersen, K.G., Makari, S., Ostenstad, S. and Potvin, R. (2016) Retreatments after Multifocal Intraocular Lens Implantation: An Analysis. Clinical Ophthalmology, 10, 365-371.

[6] Alfonso, J.F., Fernández-Vega, L., Montés-Micó, R. and Valcárcel, B. (2008) Femtosecond Laser for Residual Refractive Error Correction after Refractive Lens Exchange with Multifocal Intraocular Lens Implantation. American Journal of Ophthalmology, 146, 244-250. https://doi.org/10.1016/j.ajo.2008.03.022

[7] Muftuoglu, O., Prasher, P., Chu, C., Mootha, V.V., Verity, S.M., Cavanagh, H.D., Bowman, R.W. and McCulley, J.P. (2009) Laser in Situ Keratomileusis for Residual Refractive Errors after Apodized Diffractive Multifocal Intraocular Lens Implantation. Journal of Cataract \& Refractive Surgery, 35, 1063-1067. https://doi.org/10.1016/j.jcrs.2009.01.028

[8] Cochener, B. (2016) Discussion of Clinical Outcomes with an ERV IOL. Cataract \& Refractive Surgery Today Europe, 35-37.

[9] Weeber, H.A. and Piers, P.A. (2012) Theoretical Performance of Intraocular Lenses Correcting Both Spherical and Chromatic Aberration. Journal of Refractive Surgery, 28, 48-52. https://doi.org/10.3928/1081597X-20111103-01

[10] Artal, P., Manzanera, S., Piers, P. and Weeber, H. (2010) Visual Effect of the Combined Correction of Spherical and Longitudinal Chromatic Aberrations. Optics EXpress, 18, 1637-1648. https://doi.org/10.1364/OE.18.001637

[11] Domínguez-Vicent, A., Esteve-Taboada, J.J., Del Águila-Carrasco, A.J., FerrerBlasco, T. and Montés-Micó, R. (2016) In Vitro Optical Quality Comparison between the Mini WELL Ready Progressive Multifocal and the TECNIS Symfony. Graefe's Archive for Clinical and Experimental Ophthalmology, 254, 1387-1397. https://doi.org/10.1007/s00417-015-3240-7

[12] Esteve-Taboada, J.J., Domínguez-Vicent, A., Del Águila-Carrasco, A.J., FerrerBlasco, T. and Montés-Micó, R. (2015) Effect of Large Apertures on the Optical Quality of Three Multifocal Lenses. Journal of Refractive Surgery, 31, 666-676. https://doi.org/10.3928/1081597X-20150928-01

\section{Submit or recommend next manuscript to SCIRP and we will provide best service for you:}

Accepting pre-submission inquiries through Email, Facebook, LinkedIn, Twitter, etc. A wide selection of journals (inclusive of 9 subjects, more than 200 journals)

Providing 24-hour high-quality service

User-friendly online submission system

Fair and swift peer-review system

Efficient typesetting and proofreading procedure

Display of the result of downloads and visits, as well as the number of cited articles

Maximum dissemination of your research work

Submit your manuscript at: http://papersubmission.scirp.org/

Or contact ojoph@scirp.org 